\title{
Partially Dominant Choice
}

\author{
Georgios Gerasimou*†
}

February 19, 2015

\begin{abstract}
This paper proposes and analyzes a model of context-dependent choice with stable but incomplete preferences that is based on the idea of partial dominance: An alternative is chosen from a menu if it is not worse than anything in the menu and is also better than something else. This choice procedure provides a simple explanation of the attraction/decoy effect. It reduces to rational choice when preferences are complete in two ways that are made precise. Some preference identification and choice consistency properties associated with this model are analyzed, and certain ways in which its predictions differ from those of other recently proposed models of the attraction effect are also discussed.
\end{abstract}

Keywords: Incomplete preferences; partial dominance; attraction/decoy effect; rational choice, context dependence JEL Classification: D01, D11

\footnotetext{
*I thank Mark Dean, Takashi Hayashi, Paola Manzini, Marco Mariotti, John Quah, Michael Richter, Jörg Stoye, Christopher J. Tyson, workshop/seminar/conference audiences at Cambridge, Amsterdam, St Andrews, Queen Mary-London, EBIM 2010 (Bielefeld), ESEM 2011 (Oslo), RES 2012 (Cambridge), SIRE-BIC 2013 (Edinburgh), SAET 2013 (Paris) and AMES 2013 (Singapore) and two referees of this journal for useful comments/discussions on previous versions of the paper, the main idea of which originates in my doctoral dissertation at the University of Cambridge. The first version of the paper was titled "Choice in the Presence of Attractions" (June 2011). Financial assistance from the Scottish Institute for Research in Economics is gratefully acknowledged. Any errors are my own.

${ }^{\dagger}$ School of Economics \& Finance, University of St Andrews. Email address: gg26@st-andrews.ac.uk.
} 


\section{Introduction}

People are often faced with decision problems in which every feasible option has something superior to all others. Consumer products may have conflicting values across different, equally relevant attributes. Election candidates may have agendas or characteristics that are considered to be better in some important respects and worse in others relative to those of the competing candidates. Policy makers may be confronted with a set of feasible plans such that any two of them generate severe trade-offs. When a decision maker has no clear way of resolving such trade-offs and is unable to find a most preferred option, she could be referred to as being in decision conflict. ${ }^{1}$

Decision conflict over two alternatives can be modeled by letting the agent's preferences be incomplete, in which case the alternatives can be declared incomparable. When preferences are incomplete and a most preferred option does not exist in a given menu of feasible alternatives, it is not immediately clear how the agent does or should decide what to choose from that menu. A natural rule to consider in this case is the one whereby she chooses something feasible that is preference-undominated. This procedure has been extensively analyzed in the literature, and as shown in Sen (1971), Schwartz (1976), Moulin (1985) and Bandyopadhyay and Sengupta (1993), among others, it could result in considerably consistent behavior under the circumstances.

Despite its normative appeal, however, this benchmark model makes predictions that are often descriptively inaccurate. A case in point is the well-known attraction effect (Huber, Payne, and Puto, 1982) discussed below. The present paper's main contribution is the modification of the benchmark model in a minimal way that allows for a simple explanation of the above robust phenomenon. Specifically, the proposed model predicts that a decision maker chooses one of the undominated feasible options that are also preferred to at least one other feasible alternative. The addition of this partial dominance requirement acts as an intuitive tie breaker that helps the decision maker choose from the set of mutually incomparable undominated options. In menus where it is not satisfied, the proposed model's predictions coincide with those of the benchmark model in that any feasible option may be chosen. The consistency axioms that characterize the choice procedure are three intuitive weakenings of WARP.

A feature of the proposed model of partial dominance that is shared by the benchmark model of simple undomination is that in decision problems where the agent's preferences happen to be complete, it prescribes choice of the (single) most preferred feasible alternative. As such, it includes rational choice as a special case. The precise ways in which the model's axiomatic system must be strengthened for it to deliver the utility-maximizing choice rule are pinned down with two novel characterizations of such behavior, one that is permissive of indifference and one that is not.

The paper also attempts to answer the question of how one can recover an agent's true preferences by observing her choices when it is assumed to be known a priori that she employs the partial dominance choice procedure to make decisions. A set of conditions are given under

\footnotetext{
${ }^{1}$ This term is widely used by psychologists and consumer/marketing researchers to describe such situations; see, for instance, Shafir, Simonson, and Tversky (1993). In the philosophy of choice literature, the term "unresolved conflict" has been used synonymously by Levi (1986).
} 
which if an alternative is never chosen in the presence of some other alternative, then this fact correctly reveals a preference for the latter over the former. This revelation criterion coincides with the one proposed by Bernheim and Rangel (2009) for inferring the preferences of possibly bounded-rational decision makers from their choices in a model-free environment. The consistency properties of the proposed choice procedure are analyzed next, with reference to a large number of normative axioms that are implied by WARP. While it turns out that the procedure generally violates many of these axioms due to its context-dependent nature, some intuitive ones are shown to be consistent with it.

The paper, finally, concludes with a discussion of the related choice-theoretic literature of the attraction effect. In particular, the out-of-sample predictions of the proposed model are compared with those of other recently introduced models in four hypothetical decision problems, and some important differences between them are uncovered. Such hypothetical decision problems may be useful in future experimental work that would attempt to assess the more general empirical relevance of the various existing models of context-dependent choice.

\section{Preliminaries}

The grand set of all alternatives that the agent may be presented with is denoted $X$ and is assumed finite and with at least three elements. The collection of all nonempty subsets of $X$ is denoted $\mathcal{M}$. Each element $A$ of $\mathcal{M}$ is a menu. For two menus $A$ and $B, A \subset B$ means that $A$ is a proper submenu of $B$.

The binary relation $\succ$ captures the agent's preferences on $X$. When it is understood that $x$ is not preferred to $y, x \nsucc y$ is written. The relation $\succ$ is asymmetric if, for all $x, y \in X, x \succ y$ implies $y \nsucc x$; total or complete, if, for all $x, y \in X$, either $x \succ y$ or $y \succ x$; transitive, if, for all $x, y, z \in X, x \succ y$ and $y \succ z$ implies $x \succ z$. If all three conditions are satisfied, then $\succ$ is a strict linear order. If for all $x_{1}, x_{2}, \ldots, x_{n} \in X$ such that $x_{1} \succ x_{2} \succ x_{3} \ldots x_{n-1} \succ x_{n}$ it holds that $x_{n} \nsucc x_{1}$, then $\succ$ is acyclic. Since $X$ is finite, if $\succ$ is acyclic and complete it is also transitive and hence a strict linear order.

Two alternatives $x$ and $y$ are said to be $\succ$-incomparable if $x \nsucc y$ and $y \nsucc x$. Unless the $\succ$ incomparability relation coincides with the symmetric part of a (possibly incomplete) reflexive ${ }^{2}$ and transitive relation, two alternatives that are $\succ$-incomparable are not generally regarded as indifferent. A more suitable interpretation in such a case would be that the agent is indecisive between these alternatives. Eliaz and Ok (2006), Mandler (2009) and Gerasimou (2012b) have suggested three ways in which indifference and incomparability/indecisiveness might be distinguished based on observable choice (and in the case of the latter paper, also choice avoidance) data.

The agent's behavior is described by a choice correspondence $C: \mathcal{M} \rightarrow X$, which is a mapping satisfying $C(A) \subseteq A$ for all $A \in \mathcal{M}$. It will be assumed throughout this paper that the agent is able to find a choosable option in every menu she is presented with, and therefore $C$ is restricted to be nonempty-valued.

\footnotetext{
${ }^{2} \mathrm{~A}$ binary relation $R$ on a set $X$ is reflexive if $x R x$ holds for all $x \in X$.
} 
Before moving on it will be useful to recall the classical principles of choice consistency which are relaxed by the axioms on $C$ that are introduced below. First, $C$ satisfies the Weak Axiom of Revealed Preference (WARP) when, for any two alternatives $x$ and $y$ and menus $A$ and $B$, if $x \in C(A), y \in A \backslash C(A)$ and $x \in B$, then $y \notin C(B)$. Moreover, $C$ satisfies Property $\alpha$ if whenever $x \in C(A)$ and $x \in B \subset A$, then $x \in C(B)$. On the other hand, $C$ satisfies Property $\beta$ if whenever $x, y \in C(A), B \supset A$ and $x \in C(B)$, then $y \in C(B)$. Finally, $C$ satisfies Property $\gamma$ if whenever some option $x$ is such that $x \in C\left(A_{i}\right)$ for all $i=1, \ldots, k$ for some collection of menus $A_{1}, \ldots, A_{k}$, then $x \in C\left(\bigcup A_{i}\right)$ too (Sen, 1971).

As is well-known since Arrow (1959), WARP and nonempty-valuedness together characterize utility-maximizing behavior in the present abstract context (although the important role of the latter assumption in this result is seldom stressed). Because the aim of the paper is to present a model that is more descriptive than normative, WARP, as well as Sen's Properties $\alpha$ and $\gamma$ mentioned above, will be relaxed in various ways in what follows. The first is by means of the following axiom:

\section{$a$-WARP}

If $C(\{x, y\})=x$ and $x \in B$, then $y \notin C(B)$.

Although $a$-WARP places fewer restrictions than WARP, one could think of the two axioms as conveying the same normative message, namely that "if $x$ is revealed preferred to $y$, then $y$ is not choosable in the presence of $x$ ". The difference lies in the fact that the former does so using a narrower definition of revealed preference than the latter. According to this reasoning one would think of WARP as taking $x$ to be revealed preferred to $y$ if there is an arbitrary menu where $x$ is chosen over $y$, and of $a$-WARP as doing the same only when $x$ is chosen over $y$ in the binary menu $\{x, y\}$. Attempts at a choice-based inference concerning a preference comparison between $x$ and $y$ are not affected by the presence of alternatives other than $x$ and $y$ in the latter situation, but they are (potentially) in the former. On these grounds, one could argue that a more robust criterion on whether an agent prefers $x$ to $y$ would come from knowing that $C(\{x, y\})=x$ rather than knowing that there is some menu $A$ where $x$ is choosable and $y$ is not.

\section{b-WARP}

If $x \in C(A)$ and $y \in A \backslash C(A)$, then $y \notin C(\{x, y\})$.

While $a$-WARP requires that choice of one alternative over another in a binary menu will not be weakly reversed in a larger menu, $b$-WARP does the opposite. Yet, although the restrictions of $a$-WARP are both normatively appealing and at the same time permissive of descriptively intuitive WARP violations (see section 3.2 below), those imposed by $b$-WARP seem to retain primarily the normative component of WARP. Specifically, if one accepts that $x$ chosen over $y$ in a non-binary menu is not necessarily indicative of a clear preference for $x$ over $y$ because of the presence of other alternatives that may influence choice at that menu, then the prediction that $y$ is not choosable in $\{x, y\}$ once such a choice has been made is presumably too strong. 


\section{Observation 1}

Property $\alpha$ implies $a$-WARP. The converse is not true.

Property $\beta$ and $b$-WARP are logically distinct.

There are WARP violations that are neither $a$-WARP nor $b$-WARP violations.

The first part of the first statement is obvious. In fact, under the assumption that $C$ is nonempty-valued, $a$-WARP is equivalent to Sen's (1977) Property $\alpha 2$. The latter states that if $x \in C(A)$ and $y \in A$, then $x \in C(\{x, y\})$ and it is obvious from its name and statement that it was introduced as a weakening of Property $\alpha$. However, this property is logically distinct from both WARP and $a$-WARP if $C$ is allowed to be empty-valued. ${ }^{3}$ By contrast, $a$-WARP is weaker than WARP and more directly comparable to it than $\alpha 2$ whether nonempty-valuedness is assumed or not. As such, it admits an interpretation that is generally distinct from that of $\alpha 2$. For the second part, note that $C(\{w, x, y, z\})=y$ and $C(\{w, x, y\})=x$ are compatible with $a$-WARP but not with Property $\alpha$. The validity of the second statement is established by observing that $C(\{w, x, y\})=x$ and $C(\{x, y\})=y$ are compatible with Property $\beta$ but violate $b$-WARP, while $C(\{w, x, y\})=\{x, y\}$ and $C(\{w, x, y, z\})=x$ are compatible with $b$-WARP but not with Property $\beta$. For the last statement, note that $C(\{x, y, z\})=x$ and $C(\{w, x, z\})=y$ are permissible by both axioms but not by WARP.

\section{Proposition 1}

The following are equivalent for a nonempty-valued choice correspondence $C: \mathcal{M} \rightarrow X$ :

(a) $C$ satisfies WARP.

(b) $C$ satisfies Properties $\alpha$ and $\beta$.

(c) $C$ satisfies $a$-WARP and $b$-WARP.

(d) $C$ is rationalized by a weak order. That is, there is a unique reflexive, complete and transitive relation $\succsim$ on $X$ such that, for all $A \in \mathcal{M}$,

$$
C(A)=\{x \in A: x \succsim y \text { for all } y \in A\} .
$$

All proofs appear in Appendix A. Statement (c) of Proposition 1 provides a novel characterization of utility maximization (generally with indifference) by decomposing WARP into two distinct components in a way analogous to Sen's (1971) decomposition of WARP into Properties $\alpha$ and $\beta$ in (b). In view of the last statement of Observation 1, the decomposition is non-tautological. Both these characterizations are valid under the assumption that $C$ is nonempty-valued and defined on the full domain $\mathcal{M}$ of $X$. Compared to Sen's where the axioms place restrictions in arbitrary menus, the one above identifies a specific complementary relationship between the requirements for consistency from binary to large menus and vice versa that is both necessary and sufficient for general choice consistency to come about.

The new characterization can also be thought of as providing a novel set of normative criteria relative to which the predictions of various behavioral models of choice can be evaluated. Specif-

\footnotetext{
${ }^{3}$ See Gerasimou (2012a) for such an application of the axiom.
} 
ically, if a choice model leads to violations of both Properties $\alpha$ and $\beta$ one might be inclined to think of it as a model of severely irrational behavior. Such a model could, however, still conform with the normatively appealing $a$-WARP axiom and hence retain a substantial rationality element that might have been suppressed had Sen's characterization been the only one available.

The last axiom considered in this section imposes a behavioral restriction of a different kind:

\section{Choice Implies Rejection (CIR)}

If $A \in \mathcal{M}$ is such that $|A|>1$, then $C(A) \subset A$.

CIR is weaker than the frequently-used "single-valuedness" assumption that renders $C$ a choice function. Like that assumption, however, it adheres to a strict interpretation of the concept of choice, according to which "choosing something" coincides with "choosing something over something else". When the agent adopts it, then she chooses an alternative and rejects at least another in all menus with more than one element. Yet, it is only in binary menus where this axiom restricts choices to be single-valued.

\section{Proposition 2}

The following are equivalent for a nonempty-valued choice correspondence $C: \mathcal{M} \rightarrow X$ :

(a) $C$ satisfies CIR and WARP.

(b) $C$ satisfies $C I R$ and Property $\alpha$.

(c) $C$ satisfies CIR and a-WARP.

(d) $C$ is rationalized by a strict linear order. That is, there is a unique asymmetric, complete and transitive relation $\succ$ on $X$ such that, for all $A \in \mathcal{M}$,

$$
C(A)=\{x \in A: y \nsucc x \text { for all } y \in A\} \text {. }
$$

This special case of Proposition 1 shows that both WARP and Property $\alpha$ are equivalent to $a$-WARP for a nonempty-valued choice correspondence that satisfies CIR. In addition, it demonstrates that, in this context, either of these axiomatic systems characterizes utility-maximizing choices where no indifference ties are present. Although Propositions 1 and 2 are of some independent interest, their primary usefulness in this paper lies with their establishing the precise connections between the two benchmark models of rational choice and the behavioral model that is developed below. This is also the only result where use of the CIR axiom is made in this paper.

As already noted, the natural starting point in choice with incomplete preferences is the model where the agent is portrayed as choosing one of the preference-undominated (or maximal) elements. (An alternative $x \in A$ is undominated in this menu if there is no $y \in A$ such that $y \succ x)$. In the most general version of this model the agent's preferences are acyclic. It was shown in Sen (1971), Schwartz (1976) and Moulin (1985) that, with a full domain, this version 
is characterized by Properties $\alpha$ and $\gamma^{4}$ A characterization of this model in the case of acyclic preferences that makes it more directly comparable to this paper's main result is provided next.

\section{Proposition 3}

The following are equivalent for a nonempty-valued choice correspondence $C: \mathcal{M} \rightarrow X$ :

(a) $C$ satisfies a-WARP and Property $\gamma$.

(b) There is a unique acyclic relation $\succ$ on $X$ such that, for all $A \in \mathcal{M}$,

$$
C(A)=\{x \in A: y \nsucc x \text { for all } y \in A\} \text {. }
$$

This choice procedure obviously generalizes the models of rational choice in Propositions 1 and 2 by imposing a weaker structure on the agent's preference relation. In light of Proposition 3 and the above bibliographic remarks, it follows that if nonempty-valuedness and Property $\gamma$ hold, $a-W A R P$ and Property $\alpha$ are equivalent.

\section{Partially Dominant Choice}

\subsection{The Model}

To modify the baseline model of Proposition 3 along the lines described in the introduction, $a$-WARP is borrowed from the previous section and two new axioms that relax $b$-WARP and Property $\gamma$ are introduced.

\section{Partial $b$-WARP}

If $x \in C(A)$, then $y \notin C(\{x, y\})$ for some $y \in A \backslash C(A)$.

\section{Weak Property $\gamma$}

If $x \in C\left(A_{i}\right)$ for $i=1, \ldots, k$ and $x=C\left(A_{j}\right)$ for some $A_{j}$ with $\left|A_{j}\right|>1$, then $x \in C\left(\bigcup_{l=1}^{k} A_{l}\right)$.

It is obvious that Partial $b$-WARP relaxes $b$-WARP by requiring each choosable alternative to dominate some rather than every rejected one in the relevant binary menu. A justification on descriptive grounds for weakening $b$-WARP in this way is that when the agent chooses $x$ from $A$ it is more likely that this choice reflects a preference for $x$ over some rather than over every alternative rejected in $A$. While Partial $b$-WARP implies that every $A$-choosable alternative must be chosen over some $A$-rejected one in the relevant binary menu, the axiom is compatible with some alternative being rejected in $A$ even if no $A$-feasible alternative is chosen over it in the binary menu (this is not the case under $b$-WARP). It is worth noting that the general version of the benchmark model in (1) does not generally satisfy Partial $b$-WARP. For example, suppose $C(\{x, y\})=\{x, y\}, C(\{w, y\})=\{w, y\}, C(\{w, x\})=x$ and $C(\{w, x, y\})=\{x, y\}$. These

\footnotetext{
${ }^{4}$ Bossert, Sprumont, and Suzumura (2005) and Eliaz and Ok (2006) study aspects of this model when a weaker structure on the domain and a richer structure on preferences are assumed, respectively.
} 
choices are compatible with that model when $\succ$ is acyclic. However, although $y \in C(\{w, x, y\})$ and $w \notin C(\{w, x, y\})$, one has $w \in C(\{w, y\})$.

Regarding Weak Property $\gamma$, it clearly relaxes Property $\gamma$ by imposing fewer restrictions in the hypothesis part of the axiom. It states that it is no longer sufficient for the agent to consider some alternative to be simply choosable in each menu within a given collection for it to also be choosable in the union of the menus in this collection. Rather, such an alternative must also be uniquely choosable from one of these menus for the latter to come about.

It is noted that although Property $\gamma$ can be equivalently stated in terms of two menus (i.e. " $x \in C(A)$ and $x \in C(B)$ implies $x \in C(A \cup B)$ " is equivalent to " $x \in C\left(A_{i}\right)$ for all $i \leq k$ implies $x \in C\left(\bigcup_{i=1}^{k} A_{i}\right)$ "), this is not true for Weak Property $\gamma$. In particular, the statement " $x=C(A)$ and $x \in C(B)$ implies $x \in C(A \cup B)$ " does not generally imply the statement " $x=C\left(A_{1}\right)$ and $x \in C\left(A_{i}\right), i \neq 1$, implies $x \in C\left(\bigcup_{i=1}^{k} A_{i}\right)$ ".

\section{Proposition 4}

The following are equivalent for a nonempty-valued choice correspondence $C: \mathcal{M} \rightarrow X$ :

(a) $C$ satisfies $a$-WARP, Partial $b$-WARP, and Weak Property $\gamma \cdot{ }^{5}$

(b) There is a unique acyclic relation $\succ$ on $X$ such that, for all $A \in \mathcal{M}$,

$$
\begin{aligned}
& C(A)=A \quad \Longleftrightarrow \quad x \nsucc y \text { and } y \nsucc x \text { for all } x, y \in A
\end{aligned}
$$

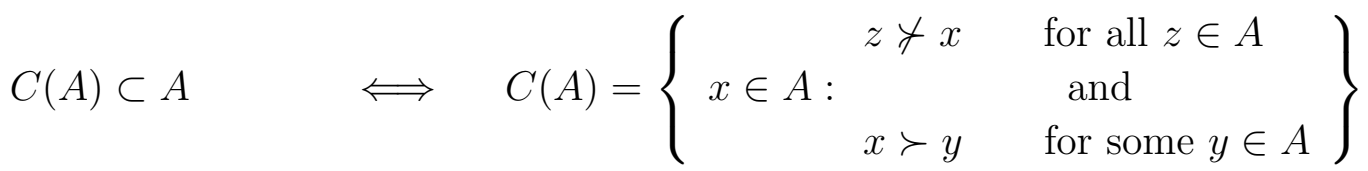

A choice correspondence $C$ that is defined by (2) will be referred to as partially dominant (PD). Moreover, if $C$ is a $\mathrm{PD}$ choice correspondence and $\succ$ is the preference relation that is associated with it in the sense of $(2)$, it will be said that $\succ$ generates $C$.

Under one interpretation of the PD model the agent chooses by following a decision rule that depends on whether the menu that she is presented with contains alternatives that are preferenceranked or not. If not, then all alternatives in the menu are mutually incomparable. Since she has been assumed to have to choose something from each menu, her decision rule in this case is to choose any feasible option, as captured in (2a). If, however, a preference comparison between some alternatives does exist, then a different decision rule is activated, following which the agent may be thought of as proceeding in two steps, and as using the same preference relation in both. In the first step, she elicits those feasible options that are undominated. (If she were to stop there, the model would coincide with the benchmark one described in (1).) In view of preference acyclicity, this set is nonempty. She then searches within this smaller set for alternatives that also dominate some other option(s) in the menu. Since at least one preference comparison is possible there by assumption, this set is also nonempty. The agent's final choice is an element of this smaller set, as captured in $(2 \mathrm{~b})$. It is worth noting that although the agent's choices as

\footnotetext{
${ }^{5}$ The logical independence of these axioms is proved in Appendix B.
} 
well as the choice rule itself are context-dependent here, her preference relation is stable across menus.

Another, simpler interpretation of the proposed choice rule is that the agent proceeds by looking at each alternative and simultaneously checking it against the two criteria required in (2b). Specifically, the agent may be thought of as rejecting all feasible options that fail to satisfy both these criteria. If at least one option exists that does satisfy them, then the agent chooses one of the options that do so. If she finds no such alternative, then everything is incomparable to everything in the menu, in which case she chooses anything.

Similar to (1), a PD choice correspondence reduces to one that is generated by utility maximization in the special case where $\succ$ is complete. Indeed, the situation of universal incomparability described in (2a) is ruled out from the outset in such a case, while for every menu $A$, an alternative $x$ in $A$ satisfies (2b) if and only if it is in fact preferred to all other alternatives in $A$. As is known from Proposition 2, the axiomatic system of Proposition 4 must be strengthened with the addition of CIR for it to deliver this model of rational choice. In that case, both Partial $b$-WARP and Weak Property $\gamma$ are implied by CIR and $a$-WARP. Despite this similarity between the acyclic-preference version of the maximal-element choice rule featured in (1) and the PD model, and despite the fact that, at any menu, the choosable options corresponding to the latter model form a subset of the set of choosable options that are derived from the former, the two models are logically distinct. Indeed, as is evident by comparing Propositions 3 and 4, both satisfy $a$-WARP but the former also satisfies Property $\gamma$ whereas the latter does not, and the latter satisfies Partial $b$-WARP which the former does not.

It has been shown in Mariotti (2008) that in the present general choice setting WARPconsistent choice can equivalently be thought of as choice that is "justified" by an asymmetric preference relation $J$ in the sense that, for every menu $A$, no two choosable alternatives in $A$ are $J$-comparable and at the same time every choosable option in that menu $J$-dominates every rejected option. The PD model retains the former but relaxes the latter "justification" requirement by merely asking for every element of $C(A)$ to $J$-dominate some element of $A \backslash C(A)$. These weaker conditions that are associated with the PD model are, however, distinct to those corresponding to the notion of a von Neumann-Morgenstern stable set in coalitional games. Indeed, the latter retains the first requirement but, unlike the PD model, also asks that every rejected alternative in $A$ is $J$-dominated by some choosable alternative in that menu.

\subsection{The Attraction Effect}

The attraction/asymmetric dominance/decoy effect is a robust empirical observation originating in the literatures of marketing research and consumer psychology, dating back to Huber, Payne, and Puto (1982). The effect challenges the descriptive accuracy of any theory of rational choice and is typically summarized as follows: When two attribute-conflicting alternatives $x$ and $y$ (e.g. alternatives that generate a price-quality trade off) are the only ones feasible, both are equally likely to be chosen. Yet, when a third option $z$ is added to the original menu, and $z$ is worse in all attributes than $y$ but also dominates $x$ and is dominated by it in some attribute, then $y$ tends to be the unique choosable option. 

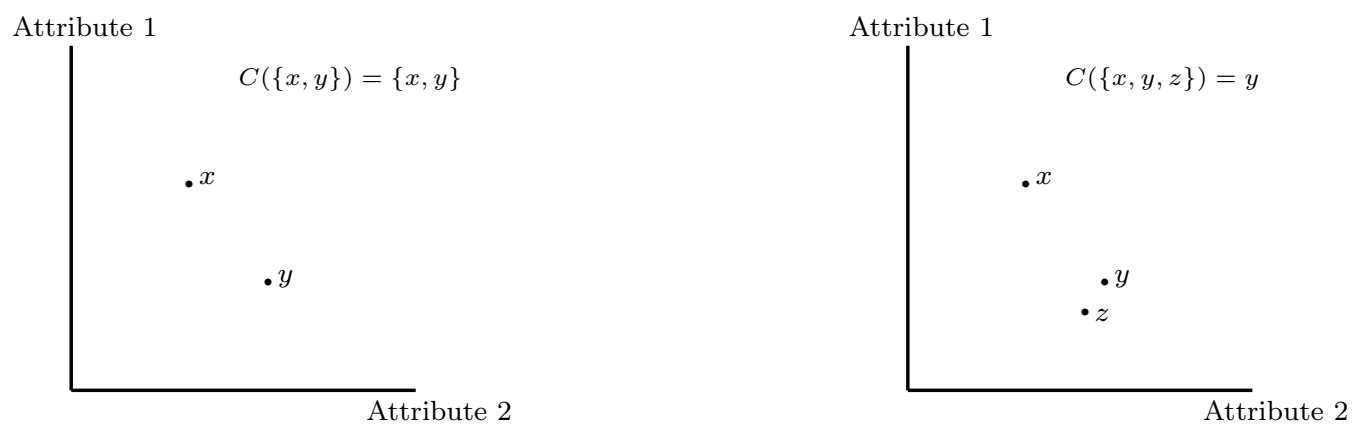

A deterministic interpretation of this pattern would have both options be choosable in the first menu, i.e. $C(\{x, y\})=\{x, y\}$, and only $y$ be choosable in the expanded menu, i.e. $C(\{x, y, z\})=$ $y$ (Fig. 1). ${ }^{6}$ Since these choices clearly violate WARP (specifically, they violate $b$-WARP and Property $\beta$ directly, and they implicitly also violate Property $\gamma$ if $C(\{x, z\})=\{x, z\})$, it readily follows that the effect lies outside the reach of any model of utility-maximizing behavior. At the same time, the choice procedure that is based on simple preference undomination is also descriptively inadequate in this regard, as it would pick both maximal elements $x$ and $y$ in menu $\{x, y, z\}$. This is not the case for the PD procedure.

\section{Observation 2}

A PD choice correspondence $C$ predicts the attraction effect when the preference relation that generates $C$ is the usual (Pareto) partial ordering on attribute space.

In addition to being able to explain the classic attraction effect, the PD model is also general enough to explain potential "idiosyncratic" attraction effects that are based on more subjective dominance relations. For instance, if the agent's incomplete preferences differ from those captured by the usual partial ordering and there are alternatives $x, y$ and $z$ where $x$ and $y$ and also $x$ and $z$ are incomparable while $y$ is preferred to $z$, an idiosyncratic attraction effect would occur if both $x$ and $y$ were choosable from the menu $\{x, y\}$ and $y$ was the unique choosable option in the menu $\{x, y, z\}$. This would also be predicted by the PD model.

In summary, the explanation of the attraction effect that is hereby suggested is that the agent has stable but incomplete preferences over attribute-conflicting alternatives and chooses among them according to the partial dominance criterion which helps her break the incomparability tie. In the absence of such a tie breaker, the agent may choose any of the feasible options. Thus, according to this model, the attraction effect cannot occur with decision makers who have a most preferred option in the pure conflict binary menu, as this option would be choosable by

\footnotetext{
${ }^{6}$ Some choice theorists (e.g. Masatlioglu, Nakajima, and Ozbay (2012) and Ok, Ortoleva, and Riella (2015)) interpret this pattern as one instance of the attraction effect, and argue that another instance is manifested in the pattern $C(\{x, y\})=x$ and $C(\{x, y, z\})=y$ (which obviously leads to a violation of $a$-WARP and hence of Property $\alpha)$. If one accepts that $C(\{x, y\})=x$ means that the agent always (or almost always) chooses $x$ and rejects $y$ at this menu, and that this agent (almost) always chooses $y$ and rejects $x, z$ at $\{x, y, z\}$, then this interpretation of the attraction effect, while possibly true for some decision makers, does not seem to be immediately supported by existing experimental data that is primarily based on between-subject designs and one-shot choices from only one of these menus for each subject. Careful experimental work that would shed light on this issue is certainly desirable.
} 
them also in the expanded trinary menu.

\subsection{Preference Revelation}

If an outside observer somehow knows that the agent's decision rule coincides with the one put forward in the PD model, is it possible for the observer to elicit the agent's (unique) preference relation by looking at her choices in various menus? Being able to answer questions of this kind is of interest, for example because the answers may provide guidance for assessing the effect that different choices of a social planner might have on a given agent's welfare. ${ }^{7}$

In the case of the PD model, if observations are available from all binary menus, then the task is trivial. ${ }^{8}$ If such observations are missing, however, it is not obvious if the above can be done. Consider two alternatives $x, y \in X$. For an outside observer to be able to conclude that the agent prefers $x$ to $y$ it is clearly necessary that, for all menus $B$, if $y \in C(B)$ holds, then $x \notin B$ also holds. It was argued in Bernheim and Rangel (2009) (BR) that this condition is a reasonable definition of revealed preference (of $x$ over $y$ ) in a model-free context, and it was also shown that the associated binary relation is generally acyclic under the full-domain assumption. Yet, in the case of the PD model this condition is not generally sufficient for accurate preference elicitation. For instance, if $X=\{w, x, y, z\}, x \succ w, x \succ z$ and incomparability prevails in all other pairs, then the agent's PD choices are $C(\{w, x, y\})=C(\{w, x, z\})=C(\{x, y, z\})=C(\{w, x, y, z\})=x$ and $C(\{w, y, z\})=\{w, y, z\}$. Although $y$ is never chosen in the presence of $x$ in this example, it is not true that this reveals preference for $x$ over $y$.

A natural question then is whether conditions exist under which the statement " $y$ is never chosen in the presence of $x$ " is true if and only if the statement " $x$ is preferred to $y$ " is true. The next result identifies some such conditions.

\section{Proposition 5}

Let $|X|=m>n>2$ and let $\mathcal{N}$ be a collection of menus that includes those with $n$ elements.

Let $C: \mathcal{N} \rightarrow X$ be a PD choice correspondence and $\succ$ the acyclic relation that generates it.

If there is a set $S$ of $n-2$ distinct alternatives in $X$ that are $\succ$-incomparable to all alternatives in $X$, then the following are equivalent for all $x, y \in X \backslash S$ :

(a) $x \succ y$.

(b) If $A \in \mathcal{N}$ and $x \in A$, then $y \notin C(A)$.

The simplest special case of the conditions provided in Proposition 5 for the BR criterion to accurately reveal the preferences of an agent who employs the PD rule is when $n=3$. In this case, the domain $\mathcal{N}$ is required to include all three-element subsets of $X$ on the one hand, while on the other hand one alternative that is incomparable to all others must also exist in

\footnotetext{
${ }^{7}$ Bernheim and Rangel (2009), Rubinstein and Salant (2012), Manzini and Mariotti (2012), Cherepanov, Feddersen, and Sandroni (2013) and Fleurbaey and Schokkaert (2013) are recent studies that are concerned with the problem of eliciting welfare preferences in models of bounded-rational or non-standard behavior. Galichon and Quah (2013) outline some recent trends in revealed preference theory more generally.

${ }^{8}$ Indeed, in this case $C(\{x, y\})=x$ would reveal $x \succ y, C(\{x, y\})=y$ would reveal $y \succ x$ and $C(\{x, y\})=\{x, y\}$ would reveal $x \nsucc y$ and $y \nsucc x$.
} 
$X$. More generally, the stated conditions feature a domain and a preference restriction, and a complementary relationship between them. Specifically, observations from all menus of size $n$, for some fixed $n>2$, must be available, and the agent's preferences must allow for $n-2$ universally incomparable alternatives to exist (this number $n-2$ can be very small relative to the number $m$ of all alternatives in $X$ ). The latter implicitly assumes that the observer is not completely agnostic about the agent's preferences. Rather, (s)he knows beforehand that such alternatives exist, and also which particular alternatives they are.

The preference restrictions are particularly demanding and limit the generality of this result. There are cases, however, where even these restrictions might be satisfied. For instance, when an experimenter is interested in knowing a subject's preferences over the main varieties of "apples" and somehow knows that the agent's choices are PD, (s)he can let the grand choice set $X$ consist of one "apple" from each main variety and also include in $X$ something that stands a good chance of being considered incomparable to all "apples", like an "orange".

\subsection{Choice (In)Consistency}

To assess the consistency properties of the PD model with regard to the agent's choice behavior across different menus, a number of intuitive axioms that are distinct from Properties $\alpha, \beta$ and $\gamma$ are now recalled. First, a choice correspondence $C$ satisfies the Aizerman axiom (Moulin, 1985) if, for all $A, B \in \mathcal{M}, C(A) \subseteq B \subseteq A$ implies $C(B) \subseteq C(A)$. It satisfies Manzini and Mariotti's (2007) Weak WARP if $\{x, y\} \subset B \subset A, x=C(\{x, y\})$ and $x=C(A)$ implies $x=C(B)$. It is consistent with Ehlers and Sprumont's (2008) Weakened WARP if, for all $A, B \in \mathcal{M}, x \in C(A)$, $y \in A \backslash C(A)$ and $y \in C(B)$ implies $x \notin B \backslash C(B)$. Finally, it satisfies the "A3" axiom proposed by Eliaz, Richter, and Rubinstein (2011) (henceforth the $E R R$ axiom) if, for all $A \in \mathcal{M}$ such that $|A| \geq 2$ and $x, y \notin A, w \in C(A \cup\{x, y\})$ holds whenever $w \in C(A \cup\{x\})$ and $w \in C(A \cup\{y\})$.

The next two concepts were introduced in Ok, Ortoleva, and Riella (2015) (OOR). A menu $A \in \mathcal{M}$ is called $C$-awkward if there is a doubleton subset $B$ of $A$ such that $C(A) \cap B \neq \emptyset$ and $C(A) \cap T \neq C(T)$. A collection of menus $\mathcal{T}$ in $\mathcal{M}$ are said to form a $C$-cover of some menu $A$ if the elements of $\mathcal{T}$ cover $A$ and $C(A) \cap T \neq \emptyset$ for all $T \in \mathcal{T}$. Now, $C$ satisfies OOR's Reference Consistency if for any $C$-awkward $A \in \mathcal{M}$ and any $C$-cover $\mathcal{T}$ of $A$, it holds that $C(T)=C(A) \cap T$ for some $T \in \mathcal{T}$. Finally, $C$ satisfies OOR's Rationality of Indifference if for any $x, y \in X$, if $\{x, y\} \subseteq C(A)$ for some $A \in \mathcal{M}$, then $C(\{x, y\})=\{x, y\}$.

\section{Observation 3}

A PD choice correspondence may violate Properties $\alpha, \beta, \gamma$, Aizerman, Weak WARP, Weakened $W A R P$ and Reference Consistency, but satisfies Rationality of Indifference and ERR.

The validity of this observation is established in Appendix A. This wealth of violations of normative axioms that is associated with the PD model may come as a surprise given that in this model the agent is actually portrayed as seeking more reasons before considering an alternative to be choosable than she does in the baseline model of (1). This search for more reasons, however, is precisely what makes the PD rule context-dependent and leads to all the above 
kinds of inconsistencies, even though it preserves rationality within each menu by ensuring that an undominated option is always chosen. The fact that a PD choice correspondence generally violates Weak WARP, in particular, shows that it cannot be formulated as a rational shortlist model à la Manzini and Mariotti $(2007)^{9}$. In addition, the fact that Reference Consistency is not satisfied in general further shows that the PD model is not a special case of the one developed in Ok, Ortoleva, and Riella (2015) either. At the same time, both these models feature choices that generally violate $a$-WARP. Therefore, both are logically distinct from the PD model.

On the other hand, the fact that PD choices are compatible with the minimal Rationality of Indifference property, even though in this model the incomparability relation between two options $x$ and $y$ that is defined by $C(\{x, y\})=\{x, y\}$ is generally intransitive, is obviously normatively appealing. The observation that the PD model is also compatible with the ERR axiom (which, as shown in Eliaz, Richter, and Rubinstein (2011), implies Property $\alpha$ whenever two alternatives are choosable in every non-singleton menu) also puts an upper bound on how irrational the behavior that is described by this model can be. It is also of interest that theories of maximal-element choice that build on (1) as well as others that satisfy many of the axioms in Observation 3 do not obey Partial $b$-WARP, and hence deviate more fundamentally from the normative $b$-WARP axiom than does the PD model. Finally, it is worth stressing that being consistent with some of the above axioms is not sufficient for a model to be free of other types of severe behavioral inconsistencies such as cyclic choices (Manzini and Mariotti, 2007; Ehlers and Sprumont, 2008), against which the PD model is robust.

\section{Related Choice-Theoretic Literature}

Three alternative explanations of the attraction effect that have been proposed in the very recent choice-theoretic literature are due to Lombardi (2009), de Clippel and Eliaz (2012) and Ok, Ortoleva, and Riella (2015). ${ }^{10}$ In Lombardi's (Lo) model the agent has acyclic preferences and chooses a preference-undominated option with the property that its lower contour set is not properly included to the lower contour set of some other undominated option. In the model of de Clippel and Eliaz (dCE) the agent has two complete strict preference orderings, calculates the pair of "scores" which reflect the number of feasible options that are inferior to a given alternative according to each ordering, and chooses one of the feasible options with the greatest minimum score. The revealed (p)reference model proposed in Ok, Ortoleva, and Riella (2015) predicts that choice from a menu is determined by the presence of reference alternatives which constrain the set of feasible options over which the agent maximizes a numerical function (interpreted as a utility function).

The "out of sample" predictions of the PD model and those of the first two models above are compared in four hypothetical decision problems over two-attribute alternatives that are shown in Fig. 2 below. The revealed (p)reference model is not included in this comparison because its

\footnotetext{
${ }^{9}$ See Apesteguia and Ballester (2013) for a recent reformulation of this model.

${ }^{10}$ In other relevant work, Kamenica (2008) studied a market model in which alternatives convey information to the consumers in ways that may induce changes in market shares that are consistent with the attraction effect. Bordallo, Gennaioli, and Shleifer (2013) studied context effects in a parametric model of choice based on a menu-dependent utility function that is linear on quality and price and is generally distorted by the relative salience of quality and price in a given menu in favour of one attribute for each good. In this special environment, that model too can explain the attraction effect.
} 
predictions depend on parameters such as which alternatives act as reference points, how the latter constrain the choosability region and the shape that the function which is to be maximized over this region takes. For the Lo and PD models, it is assumed that the preference relation is the usual partial ordering, while for the dCE model the two preference relations correspond to the natural ordering on the real line for each attribute.

Figure 2:

The predictions of three models of the attraction effect in some hypothetical problems
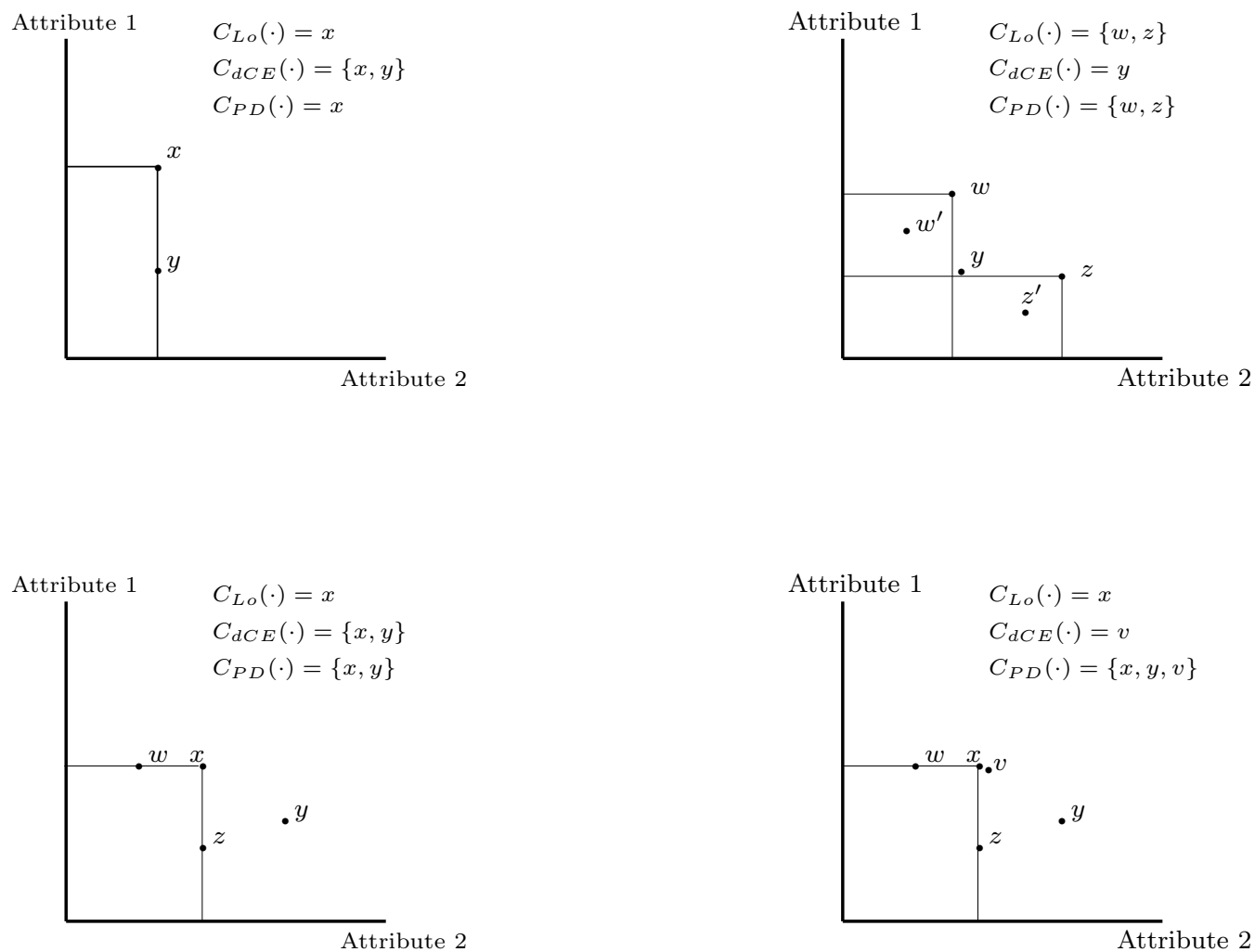

The upper left example shows that certain dominated options can be choosable under the dCE procedure. The upper right one depicts a menu where $y$ is a "compromise" between two extreme options $w^{\prime}$ and $z^{\prime}$ that are dominated by $w$ and $z$, respectively. The Lo and PD models coincide in their predictions that the "attractive" options $w$ and $z$ are the choosable ones, whereas the $\mathrm{dCE}$ procedure predicts that $y$ is uniquely chosen, just as if the menu did not include $w$ and $z$. In the latter case, unique choice of $y$ is consistent with the compromise effect (Simonson, 1989), according to which the option that provides a compromise between two extreme attributeconflicting alternatives tends to be uniquely chosen in such trinary menus. The dCE is the only model in this class that accommodates this effect with precision (although the others do not rule it out). This feature of the $\mathrm{dCE}$ model, however, is not sensitive to the positioning of the intermediate option in these menus, and any such option is predicted to still be uniquely chosen even when it cannot be thought of as a compromise one. 
In the bottom two examples, if one ignores the two dominated options $w$ and $z$ one has the two menus that generate the similarity effect, originating in Debreu (1960) and Tversky (1972). This suggests that if a binary attribute-conflicting menu is expanded with a third option such that the latter is incomparable to both but similar to only one of the original two options, then the other (now dissimilar) option of the original two tends to be uniquely chosen in the expanded menu. Neither of the above models captures the similarity effect (the PD and Lo models do not rule it out, but the dCE model does). However, these perturbed versions of the similarity-effect menus illustrate how radically different predictions the three models can generate. Specifically, the introduction of $v$ that is similar to $x$ but not to $y$ leaves the Lo model's predictions unchanged at $x$, it leads to a dramatic change in the predictions of the dCE model from $\{x, y\}$ to $v$, and leaves the PD model the only one predicting that the dissimilar option $y$ is choosable in the expanded menu, together with $x$ and $v$ that were also choosable originally.

\section{Concluding Remarks}

This paper's main contribution to the growing literature of behavioral choice theory consists of a novel choice procedure that explains the attraction/decoy effect in a simple and, one hopes, intuitive way. This procedure is based on a special kind of preference maximization that combines the core rationality criterion of preference undomination with the tie-breaking criterion of partial preference dominance. The proposed model is one of bounded-rational behavior with incomplete but menu-independent preferences, which, however, results in menu-dependent choices. In decision problems where the agent's preferences happen to be complete, the model predicts that the agent becomes a utility maximiser. The two novel characterizations of rational choice that were provided identify the precise connections between utility-maximizing behavior and what is prescribed by the model. In particular, the characterization that decomposes WARP into $a$-WARP and $b$-WARP may be of independent interest as it provides a set of criteria in addition to Sen's (1971) Properties $\alpha$ and $\beta$ relative to which the deviations from full rationality of various behavioral models can be assessed.

\section{Appendix A: Proofs}

\section{Proof of Proposition 1.}

It is well-known that $(\mathrm{a}) \Rightarrow(\mathrm{b})$ and $(\mathrm{d}) \Rightarrow(\mathrm{a})$. It will be shown that (b) $\Rightarrow(\mathrm{c}) \Rightarrow(\mathrm{d})$.

(b) $\Rightarrow$ (c). Suppose $a$-WARP is violated. Then $C(\{x, y\})=x, x \in A$ and $y \in C(A)$ for some $x, y \in X$ and $A \in \mathcal{M}$. Since $y \notin C(\{x, y\})$, this obviously violates Property $\alpha$. Thus, $a$-WARP is satisfied. Suppose $b$-WARP is not. There are $A \in \mathcal{M}$ and $x, y \in A$ such that $x \in C(A), y \in A \backslash C(A)$ and $y \in C(\{x, y\})$. Since Property $\alpha$ holds and $x \in C(A)$, it follows that $x \in C(\{x, y\})$ too. Thus, $C(\{x, y\})=\{x, y\}$. Since $A \supset\{x, y\}$ and Property $\beta$ holds, $C(\{x, y\})=\{x, y\}$ and $x \in C(A)$ implies $y \in C(A)$, a contradiction. Therefore, $b$-WARP is also satisfied.

$(\mathrm{c}) \Rightarrow(\mathrm{d})$. Define the relation $\succsim$ on $X$ by $x \succsim y$ if $x \in C(\{x, y\})$. Since all binary menus are 
included in $\mathcal{M}$ and $C$ is nonempty-valued, $\succsim$ is complete. Suppose $x \succsim y$ and $y \succsim z$ and assume to the contrary that $x \nsucceq z$. Completeness implies $z \succ x$, i.e. $C(\{x, z\})=z$. By assumption, $A:=\{x, y, z\} \in \mathcal{M}$ and $C(A) \neq \emptyset$. It follows from $a$-WARP that $x \notin C(A)$. Suppose $y \in C(A)$. Since $x \in A \backslash C(A)$ and $y \in C(A)$, it follows from $b$-WARP that $C(\{x, y\})=y$. This contradicts the postulate $x \succsim y$. Similarly, $z \notin C(A)$. Thus, $x, y, z \notin C(A)$, which is impossible. This establishes that $x \succsim z$ and therefore that $\succsim$ is also transitive, hence a weak order.

Now let $x \in C(A)$ and suppose there exists $y \in A$ such that $y \succ x$. Since $C(\{x, y\})=y$, $y \in A$ and $x \in C(A)$, this contradicts $a$-WARP. Thus, $x \succsim y$ for all $y \in A$. Conversely, suppose $x \succsim y$ for all $y \in A$ and suppose $x \notin C(A)$. It holds that $z \in C(A)$ for some $x \neq z \in A$. Since $z \in C(A), x \in A \backslash C(A)$ and $x \in C(\{x, z\})$ by assumption, $b$-WARP is contradicted. Thus, $\succsim$ rationalizes $C$. Finally, since all binary menus are included in $\mathcal{M}, \succsim$ is unique.

\section{Proof of Proposition 2.}

It is straightforward that $(\mathrm{a}) \Rightarrow(\mathrm{b}),(\mathrm{b}) \Rightarrow(\mathrm{c})$ (cf Observation 1) and (d) $\Rightarrow$ (a). It will be shown that $(\mathrm{c}) \Rightarrow(\mathrm{d})$. Suppose $C$ satisfies CIR and $a$-WARP. Define $\succ$ by $x \succ y$ if $C(\{x, y\})=x$. This is asymmetric by definition. From CIR and the full-domain assumption, it is also complete. Suppose $x \succ y, y \succ z$ and $x \nsucc z$. From CIR, $x \nsucc z$ implies $z \succ x$. Since $C$ is nonempty-valued, $C(\{x, y, z\}) \neq \emptyset$. Suppose $x \in C(\{x, y, z\})$. Since $a$-WARP holds, this contradicts the postulate $z \succ x$. Therefore, $x \notin C(\{x, y, z\})$. Similarly, $y, z \notin C(\{x, y, z\})$. Thus, $C(\{x, y, z\})=\emptyset$, a contradiction. It follows then that $x \succ z$. Thus, $\succ$ is also transitive, and hence a strict linear order.

Suppose $x \in C(A)$. It follows from $a$-WARP that $y \nsucc x$ for all $y \in A$. Now suppose the converse is true. From CIR this implies $x \succ y$ for all $y \in A \backslash\{x\}$. Suppose $x \notin C(A)$. From nonempty-valuedness, $w \in C(A)$ for some $w \in A$. Since $x \succ w$ from above, and since $a$-WARP holds, this is a contradiction. Thus, $C(A)=\{x \in A: y \nsucc x$ for all $y \in A\}$. Finally, since all binary menus are included in $\mathcal{M}, \succ$ is unique.

\section{Proof of Proposition 3.}

It is straightforward that (b) implies (a). Conversely, define $\succ$ by $x \succ y$ if $C(\{x, y\})=x$. Let $A \in \mathcal{M}$ and $x \in A$ be such that $y \nsucc x$ for all $y \in A$. From nonempty-valuedness, $x \in C(\{x, y\})$ for all $y \in A$. From Property $\gamma, x \in C(A)$. Now let $x \in C(A)$ and suppose, per contra, that $y \succ x$ for some $y \in A$. Since this is equivalent to $C(\{x, y\})=y$ and $a$-WARP holds by assumption, this is a contradiction. Finally, since all binary menus are included in $\mathcal{M}, \succ$ is unique.

\section{Proof of Proposition 4.}

Suppose there exists an acyclic relation $\succ$ on $X$ such that (2) holds. Assume, per contra, that $a$-WARP is violated. There exist $x, y \in X$ and $A \in \mathcal{M}$ such that $C(\{x, y\})=x, x \in A$ and $y \in C(A)$. From $C(\{x, y\})=x$ and (2b) it follows that $x \succ y$. Since $y \in C(A)$ it also follows from (2a) or (2b) (if $C(A)=A$ or $C(A) \subset A$, respectively) that $x \nsucc y$. This is a contradiction.

Now let $A_{1}, \ldots, A_{k} \in \mathcal{M}$ be such that $A_{1} \neq\{x\}, x=C\left(A_{1}\right)$ and $x \in C\left(A_{i}\right), 2 \leq i \leq k$. 
Define $A:=\bigcup_{i=1}^{k} A_{i}$. It is implied by (2b) and $C\left(A_{1}\right)=x$ that $z \nsucc x$ for all $z \in A_{1}$ and $x \succ y$ for some $y \in A_{1}$. Moreover, $x \in C\left(A_{i}\right)$ and (2) imply $z \nsucc x$ for all $z \in A_{i}$. Thus, $z \nsucc x$ for all $z \in A$ and $x \succ y$ for some $y \in A$. From (2b), this implies $x \in C(A)$. Hence, Weak Property $\gamma$ is satisfied.

Finally, suppose $x \in C(A)$ and there exists $z \in A \backslash C(A)$. This implies $C(A) \subset A$. Hence, (2b) and $x \in C(A)$ implies $x \succ y$ and therefore $C(\{x, y\})=x$ for some $y \in A$. From (2b) and $x \succ y$ it also follows that $y \notin C(A)$. Thus, Partial $b$-WARP is also satisfied.

Conversely, assume that $C$ is nonempty-valued and satisfies $a$-WARP, Weak Property $\gamma$ and Partial $b$-WARP. Define the asymmetric relation $\succ$ on $X$ by $x \succ y$ if $C(\{x, y\})=x$. Suppose $\succ$ is not acyclic. There exist $x_{1}, x_{2}, \ldots, x_{k} \in X$ such that $x_{1} \succ x_{2} \succ \ldots \succ x_{k} \succ x_{1}$. By assumption, $B:=\left\{x_{1}, x_{2}, \ldots, x_{k}\right\} \in \mathcal{M}$, while $C(B) \neq \emptyset$ is also true by assumption. Thus, $x_{i} \in C(B)$ for some $i \leq k$. From the $\succ$-cycle above it follows that there is $x_{j} \in B$ such that $x_{j} \succ x_{i}$, i.e. $C\left(\left\{x_{i}, x_{j}\right\}\right)=x_{j}$. This contradicts $a$-WARP.

Suppose $A \in \mathcal{M}$ is such that $x \nsucc y$ and $y \nsucc x$ for all $x, y \in A$. Let $x \in C(A)$ and $C(A) \subset A$. If $|A|=2$, then $A=\{x, y\}$ for some $y \in X$, and $x \succ y$ obviously holds, a contradiction. Suppose $|A|>2$. From Partial $b$-WARP, there exists $y \in A \backslash C(A)$ such that $x \succ y$, which is a contradiction too. It follows, therefore, that $C(A)=A$. In the other direction, let $C(A)=A$ and suppose $x \succ y$ for some $x, y \in A$. Since $y \in C(A)$ by assumption, this is a violation of $a$-WARP. This establishes (2a).

To establish (2b), let $C(A) \subset A$ for some $A \in \mathcal{M}$. Suppose $x \in C(A)$. If $z \succ x$ for some $z \in A$, then $a$-WARP is violated. Moreover, $x \in C(A), C(A) \subset A$ and Partial $b$-WARP together imply $x \succ y$ for some $y \in A \backslash C(A)$. Thus, $x \in C(A)$ and $C(A) \subset A$ implies $z \nsucc x$ for all $z \in A$ and $x \succ y$ for some $y \in A$. In the other direction, suppose there is $x \in A$ such that $z \nsucc x$ for all $z \in A$ and $x \succ y$ for some $y \in A$. This implies $C(\{x, y\})=x$ for some $y \in A$ and, since $C$ is nonempty-valued, $x \in C(\{x, z\})$ for all $z \in A$. Let $F:=\{x, y\}$ and label all other elements in $A$ by $z_{1}, \ldots, z_{k}$. Also, let $G_{i}:=\left\{x, z_{i}\right\}$. Since $x=C(F), x \in \bigcap_{i=1}^{k} C\left(G_{i}\right)$ and $\bigcup_{i=1}^{k} G_{i} \cup F=A$, it follows from Weak Property $\gamma$ that $x \in C(A)$. Finally, suppose $C(A)$ consists of all $x \in A$ with the above two properties and assume to the contrary that $C(A)=A$. Since there exist $x, y \in A$ such that $C(\{x, y\})=x$ by assumption, while $y \in C(A)=A$ also holds by assumption, $a$-WARP is violated. Hence, $C(A) \subset A$. As above, uniqueness is straightforward.

\section{Proof of Proposition 5.}

It is obviously true that (a) always implies (b). To show that the converse implication holds under the stated conditions fix an arbitrary integer $n>2$ and assume that $x \in A$ implies $y \notin C(A)$ for all $A \in \mathcal{N}$. Then, $\mathcal{N}$ includes all subsets with $n$ elements and there are $n-2$ distinct alternatives that are incomparable to everything in $X$. Let $S$ be the set of all these $n-2$ alternatives. Also, let $T=S \cup\{x, y\}$. Since $T$ has $n$ elements, it follows that $T \in \mathcal{N}$. Suppose $w \in C(T)$ for some $w \in S$. Since $w$ is universally incomparable and $C$ is $\mathrm{PD}$, the above can happen if and only if $C(T)=T$. In this case $y \in C(T)$ also holds, which contradicts (b). Thus, $w \notin C(T)$ for all $w \in S$. Moreover, since $x \in T$, it follows from (b) that $y \notin C(T)$. Hence, $C(T)=x$. Since $C(T) \subset T$, it follows from $(2 \mathrm{~b})$ that $x \succ z$ for some $z \in T$. Since $x \nsucc w$ for all 
$w \in S$ by assumption, it follows that $x \succ z$ for some $z \in T \backslash S=\{x, y\}$. Since $\succ$ is irreflexive, this implies $x \succ y$.

\section{Proof of Observation 3.}

Suppose first that $w, x, y, z$ are such that $w \succ x, y \succ z$ and no comparison is possible in all other pairs. It holds that $C(\{w, x, y, z\})=\{w, y\}$ and $C(\{w, y, z\})=y$, in violation of Property $\alpha$. If $x \succ z$ is also true, then $C(\{x, y, z\})=\{x, y\}$ and $C(\{w, x, y, z\})=\{w, y\}$, in violation of Property $\beta$. These preferences finally lead to the PD choices $C(\{w, z\})=\{w, z\}$, $C(\{w, y\})=\{w, y\}$ and $C(\{w, y, z\})=y$, in violation of Property $\gamma$.

Next, let $A=\{x, y, z\}, B=\{x, z\}$ and suppose $x \succ y$ and that all other options are incomparable. Then, $C(A)=x,\{x\} \subset B \subset A$ and $C(B)=\{x, z\}$, but $z \notin C(A)=x$, showing that Aizerman's axiom is not satisfied. Now suppose $A=\{x, y, w, z\}, B=\{x, y, w\}, w \succ y$, $x \succ y, x \succ z, z \succ w$ and all other pairs consist of incomparable options. These preferences imply $C(\{x, y\})=x=C(A)$ but $C(B)=\{x, w\}$, in violation of Weak WARP. Next, consider $A=\{w, x, y\}, B=\{x, y, z\}, x \succ w, y \succ z$ and let all other alternatives in $A, B$ be incomparable. It holds that $C(A)=x$ and $C(B)=y$, in violation of Weakened WARP.

To verify that Reference Consistency is not satisfied in general, suppose $x \succ z, w \succ y$ and that incomparability applies to all other pairs of options derived from these four. It holds that $C(\{w, x, y, z\})=\{w, x\}$. Moreover, $C(\{w, z\})=\{w, z\}$ and $C(\{x, y\})=\{x, y\}$, the menu $\{w, x, y, z\}$ is $C$-awkward, the menus $\{x, y\}$ and $\{w, z\}$ form a $C$-cover of $\{w, x, y, z\}$, and $C(\{w, x, y, z\}) \cap\{w, z\} \neq C(\{w, z\}), C(\{w, x, y, z\}) \cap\{x, y\} \neq C(\{x, y\})$.

Now suppose $x, y \in C(A)$. Since this implies $x \nsucc y$ and $y \nsucc x$, it follows that $C(\{x, y\})=$ $\{x, y\}$. Therefore, a PD $C$ satisfies Rationality of Indifference. Finally, to see that such a $C$ also conforms with ERR notice that $w \in C(A \cup\{x\})$ and $w \in C(A \cup\{y\})$ implies $z \nsucc w$ for all $z \in A \cup\{x, y\}$ and $w \succ z^{\prime}, z^{\prime \prime}$ for some $z^{\prime} \in A \cup\{x\}$ and $z^{\prime \prime} \in A \cup\{y\}$. Hence, $w \succ z$ for some $z \in A \cup\{x, y\}$, which ensures that $x \in C(A \cup\{x, y\})$.

\section{Appendix B: Axiom Independence}

The tightness of the axiomatic system of Proposition 4 is established here by means of counterexamples. Let $X=\{w, x, y, z\}$ and $\mathcal{M}=\{A: A \neq \emptyset, A \subseteq X\}$. Each of the following cases provides an example where choices satisfy all but one axiom (singletons are ignored for brevity):

\section{Not $a$-WARP}

$$
\begin{gathered}
C(\{w, x\})=w, C(\{w, y\})=\{w, y\}, C(\{w, z\})=\{w, z\}, \\
C(\{x, y\})=\{x, y\}, C(\{x, z\})=x, C(\{y, z\})=y \\
C(\{w, x, y\})=C(\{w, y, z\})=\{w, y\}, \quad C(\{w, x, z\})=\{w, x\}, C(\{x, y, z\})=\{x, y\} \\
C(X)=\{w, x, y\}
\end{gathered}
$$


Not Weak Property $\gamma$

$$
\begin{gathered}
C(\{w, x\})=w, C(\{w, y\})=\{w, y\}, C(\{w, z\})=\{w, z\}, \\
C(\{x, y\})=\{x, y\}, C(\{x, z\})=x, C(\{y, z\})=y \\
C(\{w, x, y\})=C(\{w, y, z\})=C(\{w, x, z\})=w, C(\{x, y, z\})=y \\
C(X)=w
\end{gathered}
$$

Not Partial $b$-WARP

$$
\begin{gathered}
C(\{w, x\})=w, C(\{w, y\})=\{w, y\}, C(\{w, z\})=\{w, z\}, \\
C(\{x, y\})=\{x, y\}, C(\{x, z\})=x, C(\{y, z\})=\{y, z\} \\
C(\{w, x, y\})=C(\{w, y, z\})=\{w, y\}, C(\{w, x, z\})=w, \quad C(\{x, y, z\})=y \\
C(X)=\{w, y\}
\end{gathered}
$$

\section{References}

Apesteguia, J., and M. Ballester (2013): "Choice by Sequential Procedures," Games and Economic Behavior, 77, 90-99.

Arrow, K. J. (1959): "Rational Choice Functions and Orderings," Economica, 26, 121-127.

Bandyopadhyay, T., and K. Sengupta (1993): "Characterization of Generalized Weak Orders and Revealed Preference," Economic Theory, 3, 571-576.

Bernheim, B. D., and A. Rangel (2009): "Beyond Revealed Preference: Choice-Theoretic Foundations for Behavioral Welfare Economics," Quarterly Journal of Economics, 124, 51-104.

Bordallo, P., N. Gennaioli, and A. Shleifer (2013): "Salience and Consumer Choice," Journal of Political Economy, 121, 803-843.

Bossert, W., Y. Sprumont, and K. Suzumura (2005): "Maximal-Element Rationalizability," Theory and Decision, 58, 325-350.

Cherepanov, V., T. Feddersen, and A. Sandroni (2013): "Revealed Preferences and Aspirations in Warm Glow Theory," Economic Theory, 54, 501-535.

de Clippel, G., and K. Eliaz (2012): "Reason-Based Choice: A Bargaining Rationale for the Attraction and Compromise Effects," Theoretical Economics, 7, 125-162.

Debreu, G. (1960): "Review of R. D. Luce, Individual Choice Behavior: A Theoretical Analysis," American Economic Review, 50, 186-188.

Ehlers, L., and Y. Sprumont (2008): "Weakened WARP and Top-Cycle Choice Rules," Journal of Mathematical Economics, 44, 87-94. 
Eliaz, K., and E. A. OK (2006): "Indifference or Indecisiveness? Choice-theoretic Foundations of Incomplete Preferences," Games and Economic Behavior, 56, 61-86.

Eliaz, K., M. Richter, and A. Rubinstein (2011): "Choosing the Two Finalists," Economic Theory, 46, 211-219.

Fleurbaey, M., and E. Schokkaert (2013): "Behavioral Welfare Economics and Redistribution," American Economic Journal: Microeconomics, 5, 180-215.

Galichon, A., and J. K.-H. Quah (2013): "Symposium on Revealed Preference Analysis," Economic Theory, 54, 419-423.

Gerasimou, G. (2012a): "Asymmetric Dominance, Deferral and Status Quo Bias in a Theory of Choice with Incomplete Preferences," MPRA Working Paper $4009 \%$.

(2012b): "Incomplete Preferences and Rational Choice Avoidance," mimeo.

Huber, J., J. W. Payne, and C. Puto (1982): "Adding Asymmetrically Dominated Alternatives: Violations of Regularity and the Similarity Hypothesis," Journal of Consumer Research, 9, 90-98.

KamenicA, E. (2008): "Contextual Inference in Markets: On the Informational Content of Product Lines," American Economic Review, 98, 2127-2149.

Levi, I. (1986): Hard Choices: Decision Making under Unresolved Conflict. Cambridge: Cambridge University Press.

Lombardi, M. (2009): "Reason-Based Choice Correspondences," Mathematical Social Sciences, $57,58-66$.

Mandler, M. (2009): "Indifference and Incompleteness Distinguished by Rational Trade," Games and Economic Behavior, 67, 300-314.

Manzini, P., and M. Mariotti (2007): "Sequentially Rationalizable Choice," American Economic Review, 97, 1824-1839.

- (2012): "Categorize then Choose: Boundedly Rational Choice and Welfare," Journal of the European Economic Association, 10, 1141-1165.

Mariotti, M. (2008): "What Kind of Preference Maximization Does the Weak Axiom of Revealed Preference Characterize?," Economic Theory, 35, 403-406.

Masatlioglu, Y., D. Nakajima, and E. Y. Ozbay (2012): "Revealed Attention," American Economic Review, 102, 2183-2205.

Moulin, H. (1985): "Choice Functions over a Finite Set: A Summary," Social Choice and Welfare, 2, 147-160.

Ok, E. A., P. Ortoleva, and G. Riella (2015): "Revealed (P)reference Theory," American Economic Review, 105, 299-321. 
Rubinstein, A., and Y. Salant (2012): "Eliciting Welfare Preferences from Behavioural Datasets," Review of Economic Studies, 79, 375-387.

Schwartz, T. (1976): "Choice Functions, "Rationality" Conditions, and Variations of the Weak Axiom of Revealed Preference," Journal of Economic Theory, 13, 414-427.

SEn, A. (1971): "Choice Functions and Revealed Preference," Review of Economic Studies, 38, $307-317$.

(1977): "Social Choice Theory: A Re-Examination," Econometrica, 45, 53-89.

Shafir, E., I. Simonson, and A. Tversky (1993): "Reason-Based Choice," Cognition, 11, $11-36$.

Simonson, I. (1989): "Choice Based on Reasons: The Case of Attraction and Compromise Effects," Journal of Consumer Research, 16, 158-174.

Tversky, A. (1972): "Elimination by Aspects: A Theory of Choice," Psychological Review, 79, 281-299. 